\title{
Static and Dynamic Balance Disorders in Patients With Rheumatoid Arthritis and Relationships With Lower Extremity Function and Deformities: A Prospective Controlled Study
}

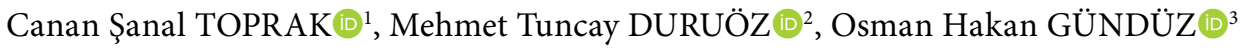 \\ ${ }^{1}$ Department of Physical Medicine and Rehabilitation, Marmara University School of Medicine, Istanbul, Turkey \\ ${ }^{2}$ Department of Physical Medicine and Rehabilitation, Division of Rheumatology, Marmara University School of Medicine, İstanbul, Turkey \\ ${ }^{3}$ Department of Physical Medicine and Rehabilitation, Division of Algology, Marmara University School of Medicine, Istanbul, Turkey
}

\begin{abstract}
Objectives: This study aims to evaluate the static and dynamic balance disorders of patients with rheumatoid arthritis (RA) and to disclose the relationships with clinical, functional, and radiological findings of lower extremities.

Patients and methods: A total of 81 patients with RA (15 males, 66 females; mean age $48.9 \pm 10.4$ years; range 22 to 67 years) were compared with 84 age and sex-matched healthy controls (14 males, 70 females; mean age $45.9 \pm 12.1$ years; range 24 to 70 years). Radiographic assessments of feet were performed to evaluate the presence of pes planus, hallux valgus, metatarsus primus varus, and splaying foot deformities. Foot functions of patients were determined with Foot and Ankle Outcome Score. The balance disorders of the subjects were evaluated with three static (modified clinical test of sensory interaction and balance, unilateral stance, weight bearing squat) and three dynamic (step-up-and-over, sit-to-stand, tandem walk) balance tests via the 'Neurocom Balance Master' device.

Results: Rheumatoid arthritis patients had significantly higher sway velocity in unilateral stance and modified clinical test of sensory interaction and balance tests, higher step width and lower speed when walking on a line, lower rising index and higher movement time in step-up-and-over test compared to healthy controls $(p<0.05)$. Performances on the sit-to-stand and weight bearing squat tests were comparable between both groups. Of the patients, although $61 \%$ had hallux valgus, $52 \%$ had metatarsus primus varus, $33 \%$ had pes planus, and $26 \%$ had splaying foot, these deformities were not correlated with Foot and Ankle Outcome Score or balance disorders. Presence of swollen joint was determined as the most relevant factor for balance disorders of RA patients.

Conclusion: Patients with RA may have increased risk for balance disorders due to cumulative effect of the lower extremity impairments seen in the course of disease.

Keywords: Balance disorder; foot deformity; functional status; rheumatoid arthritis.
\end{abstract}

Balance, a complex function involving several neuromuscular processes, is an ability of the body's center of gravity (COG) to be controlled with minimum postural sway and maximum stability within the base of support. ${ }^{1}$ Balance is classified into two groups as static and dynamic balance. ${ }^{2}$ While static balance can keep the body's COG within the base of support, dynamic balance is the ability to move the COG in a controlled manner within the base of support.
In patients with rheumatoid arthritis (RA), balance can be impaired because of impaired joint proprioception secondary to foot deformities and arthritis seen in lower extremities (LEs), muscle weakness and limitation of joint movements, or central nervous system impairment.

Previous studies have shown that RA causes impaired static and dynamic balance that is associated with age and Body Mass Index (BMI). ${ }^{2-4}$ On the other hand, $\mathrm{RA}$ is also

Received: November 07, 2017 Accepted: December 09, 2017 Published online: January 15, 2018

Correspondence: Canan Şanal Toprak, MD. Marmara Üniversitesi Pendik Eğitim ve Araştırma Hastanesi Fiziksel Tıp ve Rehabilitasyon Anabilim Dalı, 34899 Pendik, İstanbul, Turkey. Tel: +90 533 - 1381032 e-mail: canansanal@hotmail.com

\section{Citation:}

Şanal Toprak C, Duruöz MT, Gündüz OH. Static and Dynamic Balance Disorders in Patients With Rheumatoid Arthritis and Relationships With Lower Extremity Function and Deformities: A Prospective Controlled Study. Arch Rheumatol 2018;33(3):328-334. 
associated with increased risk for falling. ${ }^{3,5-9}$ Pain, morning stiffness, joint instability, deformities in weight-bearing joints, limitation of joint motion, and deterioration in joint proprioception have been shown to reduce postural control. ${ }^{10}$ As falls and balance disorders are more common during dynamic activities such as walking and stair climbing, it is important to evaluate both dynamic and static balance. Studies evaluating dynamic balance in RA are limited, and existing evaluations have generally been conducted as observational tests without using a balance device. Static balance studies tend to evaluate postural sway in the standing posture with closed and open eyes; ${ }^{3,11}$ however, these studies are usually performed with insufficient patient numbers or without control groups. In addition, although risk factors for the fear of falling or fall incidence were studied by many authors, ${ }^{12-16}$ studies about the factors that might be associated with the balance parameters are insufficient. In a previous study, authors emphasized that further investigations into risk factors such as foot and ankle characteristics associated with balance parameter in RA patients are required. ${ }^{2}$

To the best of our knowledge, there is no report about the relationship between the radiographic foot deformities and dynamic balance parameters, although the effects of foot deformities on functional ambulation or fall incidence were evaluated subjectively using clinical observational tests or outcome measurement scales. .,17,18 Therefore, in this study, we aimed to evaluate the static and dynamic balance disorders of patients with RA and to disclose the relationships with clinical, functional, and radiological findings of lower extremities.

\section{PATIENTS AND METHODS}

A total of 81 patients ( 15 males, 66 females; mean age $48.9 \pm 10.4$ years; range 22 to 67 years) who were admitted to the rheumatological disorders outpatient clinic of the Physical Medicine and Rehabilitation Department between April 2014 and April 2015 and 84 age and sex-matched healthy controls (14 males, 70 females; mean age $45.9 \pm 12.1$ years; range 24 to 70 years) without any pathology in the musculoskeletal system were included in this prospective controlled study. A priori sample size calculation based on previous studies about the balance disorders in RA patients ${ }^{2,19}$ indicated that 30 participants for each group were needed for this study with a power of 0.80 . The study protocol was approved by the Marmara University Hospital Ethics Committee under the number 70737436050.06.04-1400057258. A written informed consent was obtained from each patient. The study was conducted in accordance with the principles of the Declaration of Helsinki.

The study criteria required patients diagnosed with RA according to American College of Rheumatology 2010 diagnosis criteria and aged 18-70. Patients were excluded if they were diagnosed with any neurological or metabolic disease that may cause balance disorder, diabetes mellitus, major depression, polyneuropathy or sensory deficit, any contracture or range of motion limitations of LEs, length difference between the LEs of more than $1 \mathrm{~cm}$, any surgical intervention of the LEs or vertebrae-related problems, scoliosis or kyphosis, visual or vestibular disorder, or LEs muscle strength under $4 / 5$.

All participants were evaluated using a form comprising demographic data such as age, height, weight and educational status. The range of joint motion, muscle strength of the LEs and any deformities were investigated, and any painful or swollen joints found in the LEs were recorded. Disease activity score 28 (DAS28) was calculated to indicate the disease activity.

Anteroposterior and lateral weight bearing foot radiographs that were taken for follow-up in the last year were assessed to evaluate foot deformities. Pes planus (PP) was assessed by measuring the angle between the tangent line drawn from the lower face of the calcaneus and the line drawn from the lower face of the calcaneus to the base of first metatarsal in lateral $\mathrm{X}$-ray views. An angle smaller than 18 degrees was accepted as PP.

In anteroposterior X-ray views, metatarsus primus varus (MPV), hallux valgus (HV), and splaying foot (SF) were assessed by measuring the angle between the longitudinal axis of the first and second metatarsus (M1/2), the first metatarsus and first phalanges (M1/P1), and the first and fifth metatarsus (M1/5), respectively. An M1/2 angle of more than 9, an M1/P1 angle 
of more than 15 , and an M1/5 angle of more than 35 were accepted as MPV, HV, and SF, respectively. The measurements were performed by the same author, blinded to patients' details and to other measurements.

The Foot and Ankle Outcome Score (FAOS), which assesses pain; symptoms involving stiffness, swelling, and range of motion; function in daily living, sports, and recreation; and foot and ankle-related quality of life, was applied to assess patients' foot function.

The static and dynamic balance parameters of the patient and control groups were evaluated using the NeuroCom Balance Master (NeuroCom International Inc., Clackamas, Oregon, USA), which is a computerized device that is used to evaluate static and dynamic balance disorders. All balance assessments were repeated three times by the same author.

\section{Static balance assessments}

For weight bearing squat (WBS), the participant stands on a firm surface with eyes open and attempts to maintain equal weight on each leg while standing at $0,30,60$, and 90 degrees of knee flexion. In all these positions, the percentage of body weight borne by each leg is measured. The difference in percentage between the right and left leg is considered normal up to $7 \%$.

Modified clinical test of sensory interaction and balance assesses the participant's postural sway velocity (SV) (degrees/second) under sensory conditions. The participant stands on a firm surface and performs some procedures first with eyes open and then with eyes closed. The participant repeats the same procedures on a foam floor.

In unilateral stance, the participant stands on one foot, first with eyes open and then with eyes closed. The procedure is repeated with the participant standing on the other foot. This test quantifies the participant's postural SV (degrees/second).

\section{Dynamic balance assessments}

The tandem walk (TW) quantifies characteristics of gait as the participant walks heel to toe on a black line in the middle of the force plate. The measured parameters are step width $(\mathrm{cm})$, speed $(\mathrm{cm} /$ second), and endpoint SV (degree/second).
In step-up-and-over (SUO), the participants are asked to step up onto a curb placed on the force plate with one foot, lift the body through an erect standing position over the box, swing the other foot over the box, and lower the foot to land the swing leg on the force plate. The measured parameters are the rising index (force to rise), movement time, and impact index (control of impact force descending onto the swing leg).

For sit-to-stand (STS), the participants are asked to rise from a seated position to a standing position on the force plate and maintain the centered COG position. The measured parameters are weight transfer time, rising index (force exerted to rise), SV during the rising phase, and left/right symmetry of the rising force.

\section{Statistical analysis}

The data obtained in this study were evaluated using IBM SPSS version 22.0 software (IBM, Corp., Armonk, NY, USA). The normality of the distribution of parameters was evaluated using the Shapiro-Wilks test. In addition to descriptive statistical methods (mean, standard deviation), the Student's t-test was used to compare two groups with normal distribution of quantitative data, and the Mann-Whitney $\mathrm{U}$ test was used to compare two groups with unevenly distributed parameters. The paired sample t-test was used for an intra-group comparison of the parameters with normal distribution, and the Wilcoxon signed-rank test was used to compare the parameters with uneven distribution. The qualitative data were compared using Chi-square, Yates' correction for continuity, and McNemar's tests. Pearson correlation analysis was used to evaluate the interactions between the parameters with a normal distribution. $P$ values below 0.05 were considered statistically significant.

\section{RESULTS}

Age, sex, and BMI characteristics were similar for patient and control groups $(p<0.05)$. The mean disease duration of patients was 76.0 \pm 77.0 months and the mean DAS28 was $3.7 \pm 1.2$.

For WBS test, no statistically significant differences were found between the groups ( $p>0.05$ ), and weight bearing distribution through each LE was not significantly different between 
Table 1. Comparison of sway velocities of patients and healthy controls

\begin{tabular}{|c|c|c|c|c|c|}
\hline & \multicolumn{2}{|c|}{ Patients } & \multicolumn{2}{|c|}{ Controls } & \multirow[b]{2}{*}{$p$} \\
\hline & Mean \pm SD & Median & Mean \pm SD & Median & \\
\hline MCTSIB-Firm surface-eyes open & $0.3 \pm 0.3$ & 0.3 & $0.3 \pm 0.1$ & 0.26 & 0.293 \\
\hline MCTSIB-Firm surface-eyes closed & $0.4 \pm 0.2$ & 0.36 & $0.4 \pm 0.3$ & 0.33 & 0.193 \\
\hline MCTSIB-Foam surface-eyes open & $1.0 \pm 0.4$ & 0.96 & $0.8 \pm 0.2$ & 0.73 & $0.001^{* *}$ \\
\hline MCTSIB-Foam surface-eyes closed & $1.7 \pm 0.5$ & 1.6 & $1.4 \pm 0.3$ & 1.33 & $0.001^{* *}$ \\
\hline US-left-eyes open & $1.2 \pm 0.6$ & 1.06 & $0.9 \pm 0.2$ & 0.86 & $0.001^{* *}$ \\
\hline US-left-eyes closed & $2.5 \pm 1.7$ & 2.03 & $1.9 \pm 0.5$ & 1.76 & $0.001^{* *}$ \\
\hline US-right-eyes open & $1.2 \pm 0.4$ & 1.1 & $1.0 \pm 0.2$ & 0.93 & $0.001^{* *}$ \\
\hline US-right-eyes closed & $2.5 \pm 1.7$ & 2.06 & $1.9 \pm 0.6$ & 1.78 & $0.007^{* * *}$ \\
\hline
\end{tabular}

the groups for the 30,60, and 90 degrees of flexion ( $p>0.05)$.

Regarding the patients' postural SV (degrees/ second) under sensory conditions on a firm surface, no significant difference was evident in the mean velocity between the groups ( $p>0.05)$. However, the control group had significantly higher mean levels when the test was performed on a foam surface with eyes closed or open $(p<0.01)$. For the unilateral stance, the patient group had a significantly higher mean SV compared with the control group $(p<0.01)$. The patient group also had a significantly higher mean SV while standing on the right foot with eyes closed or open $(p<0.01)$ (Table 1$)$.

The results of TW test showed that the patient group had a significantly higher mean step width compared with the control group $(p<0.05)$. The mean speed was significantly lower in the patient group than in the control group $(p<0.01)$, but no statistically significant difference was detected between the groups in terms of the mean endpoint SV ( $p>0.05)$.

Regarding SUO test with both feet, the mean rising index was significantly lower $(p<0.01)$ and the mean duration was significantly higher in the patient group than in the control group $(p<0.01)$. However, the mean impact index with both feet was not significantly different between groups ( $p>0.05)$. The results of the STS test showed no significant difference between the groups in the mean measures of any of the parameters ( $p>0.05)$.

The impacts of demographic and clinical factors on the balance parameters were evaluated for the patient group. No significant correlation was found between the demographic characteristics and balance parameters $(p<0.05)$. Swollen joints

Table 2. Correlation between clinical features and balance parameters

\begin{tabular}{|c|c|c|c|c|c|c|}
\hline & \multicolumn{2}{|c|}{ LE swollen joint numbers } & \multicolumn{2}{|c|}{ LEs tender joint numbers } & \multicolumn{2}{|c|}{ DAS28 } \\
\hline & $\mathrm{r}$ & $p$ & $\mathrm{r}$ & $p$ & $\mathrm{r}$ & $p$ \\
\hline TW-step width (cm) & 0.130 & 0.250 & 0.038 & 0.735 & -0.048 & 0.671 \\
\hline TW-speed $(\mathrm{cm} / \mathrm{sec})$ & 0.056 & 0.622 & -0.025 & 0.825 & -0.229 & $0.041^{*}$ \\
\hline MTCSIB-foam surface-eyes open (degrees/sec) & 0.540 & $0.001^{* *}$ & 0.264 & $0.017^{*}$ & 0.169 & 0.131 \\
\hline MTCSIB-foam surface-eyes closed (degrees/sec) & 0.127 & 0.259 & 0.045 & 0.692 & 0.037 & 0.743 \\
\hline US-left-eyes open (degrees/sec) & 0.402 & $0.001^{* *}$ & 0.093 & 0.410 & 0.067 & 0.553 \\
\hline US-left-eyes closed (degrees/sec) & 0.346 & $0.002^{* * *}$ & 0.067 & 0.555 & 0.086 & 0.446 \\
\hline US-right-eyes open (degrees/sec) & 0.556 & $0.001^{* *}$ & 0.259 & $0.020^{*}$ & 0.228 & $0.040^{*}$ \\
\hline US-right-eyes closed (degrees/sec) & 0.373 & $0.001^{* * *}$ & 0.068 & 0.547 & 0.137 & 0.224 \\
\hline SUO-left rising index (\%) & -0.231 & $0.038^{*}$ & -0.146 & 0.194 & -0.168 & 0.134 \\
\hline SUO-left movement time (sec) & 0.482 & $0.001^{* *}$ & 0.248 & 0.186 & 0.224 & $0.044^{*}$ \\
\hline SUO-left impact index (\%) & 0.010 & 0.932 & 0.164 & 0.145 & 0.125 & 0.267 \\
\hline SUO-right rising index (\%) & -0.130 & 0.249 & -0.060 & 0.596 & -0.189 & 0.091 \\
\hline SUO-right movement time (sec) & 0.397 & $0.001^{* * *}$ & 0.131 & 0.243 & 0.191 & 0.087 \\
\hline SUO-right impact index (\%) & 0.094 & 0.403 & 0.210 & 0.060 & 0.156 & 0.165 \\
\hline
\end{tabular}


of LEs were found to be the most relevant factors on balance. Table 2 presents the relationship between the clinical factors and the balance parameters.

Anteroposterior plain radiography was obtained for 76 patients and lateral plain radiography for 78 patients. Of those patients, 73 (92.5\%) had evidence of at least one foot deformity, HV being the most common. Sixty-one patients had HV (80.2\%), 52 had MPV (68.4\%), 26 had SF (35.5\%), and 33 had PP (42.3\%).

No significant difference was found between the deformities of the left and right feet ( $p>0.05$ ), and the presence of a deformity did not have any significant impact on the SV ( $p>0.05)$.

While the FAOSs were reported to have a negative correlation with the number of swollen or tender joints in the LEs, no significant relationship was evident between the foot deformities and FAOSs. Additionally, the mean FAOSs of patients with right or left metatarsalgia were significantly lower compared with those of patients free of metatarsalgia symptoms $(p<0.01)$. Overall, a correlation was not evident between the FAOSs and balance parameters ( $p>0.05$ ).

\section{DISCUSSION}

This study evaluated the clinical, functional, and radiological factors that might impact balance while analyzing the static and dynamic balance of patients diagnosed with RA. To the best of authors' knowledge, this study has recruited more patients and controls than previous studies focusing on this subject. Since previous studies have reported that age, sex, and BMI could impact balance, ${ }^{2-4}$ participants with similar characteristics for patient and control groups were selected to be included in this study.

During the WBS test, the measures of weight transferred to the right or left LE were insignificant when compared with the control group. This finding might be because of the symmetrical nature of RA and because of the similarity of the left and right sides with regards to swollen or tender joints of the LEs or foot deformities in patient group. Asymmetrical distribution of weight through the LEs found in both the patient and control groups might be due to the dominant foot entity. A previous study demonstrated that foot dominance and postural control have a correlation, ${ }^{20}$ and this finding is consistent with the data of this study.

This study reported that the patient group had a significantly higher mean SV compared with the control group. This finding supports those of previous studies, ${ }^{2-4}$ which stated that an increased SV could indicate a high risk of falls. ${ }^{2,3,19}$ The higher SV in patient group might also suggest an increased risk of fall incidences. As it was expected, when evaluating the correlation between SV and the clinical parameters, the number of swollen joints in the LEs was significantly related with SV. In accordance with findings of this study, a previous study reported that increased inflammation in the LEs could deteriorate proprioceptive responses and thereby increase the SV. ${ }^{21}$

This study showed no significant difference evident among any of the parameters for the patient and control groups during the STS test, which met the expectations. A previous study, which conducted the STS test with $135 \mathrm{RA}$ patients, reported a strong correlation with the strength of the extensors of the knee joint. ${ }^{22}$ While this test is used to evaluate the strength of the muscles and symmetry, results in the normal ranges are not surprising because the patients in this study had symmetrical joint involvement, and those with muscle weakness were excluded.

Tandem walk is a more specific test, compared with walking, for evaluating the disturbances of balance. This test requires a more delicate control of the COG and the ability to rebuild the stabilization on a narrow support ground. In previous studies, disturbances in TW and the tandem standing test were reported to be correlated with falling incidences. ${ }^{11,23}$ As mentioned above, an increased $\mathrm{SV}$ is an indicator of increased risk of falls and, in accordance with this finding, a decreased velocity and increased step width were reported in the TW test in this study. The reason why no significant differences were found between the groups in the endpoint SV could be that the patients managed to control the sway behavior because they walked rather slowly with big steps, and they concentrated on their motor actions. This is a good example of the compensatory strategies that patients develop to cope with the disturbances of balance and avoid falls. 
The SUO is a complex action that has a prominent role in everyday activities and pain, fatigue, biomechanical disturbances and muscle weakness may affect this ability. In this study, the time needed to perform SUO test was prolonged, and the rising index was significantly decreased in the patient group than in the control group. Previous studies reported that RA patients have some difficulties to perform the SUO test. ${ }^{5,6,23}$ In this study, the prolonged time needed for SUO test was shown to be correlated with the number of swollen joints.

In this study, the relationship between foot deformities and balance was also investigated. Sixty-one patients had HV, 52 had MPV, 26 had SF, and 33 had PP. In a previous study, which investigated foot deformities in Turkish RA patients $(n=40), 78.8 \%$ of the patient population reported foot deformity, and the frequency and sequence of the deformities were similar to those reported in this study. Although somewhat weak, the previous study reported a correlation between the FAOSs and foot deformities; ${ }^{17}$ however, such a correlation was not found in this study. While a correlation between the FAOSs and balance parameters was not found, a relationship was evident between FAOSs and the number of swollen/tender joints at the LEs or metatarsalgia. This finding emphasizes that the number of swollen or tender joints at the LEs is the most important factor affecting balance. Similarly, a previous study stated that pain involving LEs has an impact on functional ambulation; however, this study reported no evidence of a correlation between foot deformities and functional ambulation. ${ }^{18}$ Another study reported that while the risk of falls increases with the number of swollen joints, foot deformities do not affect this risk. $^{5}$ The reason why foot deformities do not significantly impair the functionality might be that patients learn how to cope with these problems and develop compensatory strategies, particularly when the chronic nature of RA is considered. However, when pain complicates the situation, patients' coping strategies fail to compensate the ongoing process.

The assessment of both static and dynamic balance using NeuroCom Balance Master is one of the strengths of this study. NeuroCom Balance Master assessment protocols quantify the effect of impairments on a patient's ability to carry out mobility tasks required for safe and effective daily life functions and help to accurately identify the sensory and motor impairments underlying a patient's functional limitations and disabilities by providing objective information. ${ }^{24}$ In this study, the impaired parameters of each balance task have been reported clearly. These data can be used to better define the goals of an effective therapy program and assess the effectiveness of the intervention in clinical practice and also provide valuable insight for further investigations into management of balance disorders of RA patients.

The other strengths of this study are having a higher number of patients and healthy controls compared with previous studies on similar topics and evaluating possible correlations between the balance parameters and various functional, clinical, and radiological entities of LEs. The results highlighted that foot functions and deformities are not directly correlated with balance disorder in RA patients and most relevant factor on balance is swollen LE joints. Thus, these findings may lead the clinicians to consider that the impaired balance of patients may occur as an independent result of RA probably due to cumulative effect of the LEs impairments and arthritis seen in the course of disease may augment the existing balance disorder.

This study has some limitations that should be pointed out. Firstly, fear of falling and history of fall among patients were not assessed. However, previous studies have already shown that increased $\mathrm{SV}$ is a strong predictor of increased risk and fear of falling. ${ }^{2,3,19}$ Secondly, while patients diagnosed with any neurological or metabolic disease or major depression were excluded, cognitive function, which might affect balance of patients, was not evaluated directly in this study.

In conclusion, the results of this study showed that patients with RA may have increased risk of balance disorders due to cumulative effect of the LEs impairments seen in the course of disease. Balance disorder causes inactivity and sedentary life, which may lead to some secondary effects such as increased risk of falls, osteoporosis, fracture, and cardiovascular disease. Therefore, based on the results of this study, further investigations are required into management of balance disorder in RA patients such as proprioceptive training, 
balance exercises and rehabilitation, and assistive devices for lower extremities.

\section{Declaration of conflicting interests}

The authors declared no conflicts of interest with respect to the authorship and/or publication of this article.

\section{Funding}

The authors received no financial support for the research and/or authorship of this article.

\section{REFERENCES}

1. Shumway-Cook A, Anson D, Haller S. Postural sway biofeedback: its effect on reestablishing stance stability in hemiplegic patients. Arch Phys Med Rehabil 1988;69:395-400.

2. Rome K, Dixon J, Gray M, Woodley R. Evaluation of static and dynamic postural stability in established rheumatoid arthritis: exploratory study. Clin Biomech (Bristol, Avon) 2009;24:524-6.

3. Aydoğ E, Bal A, Aydoğ ST, Cakci A. Evaluation of dynamic postural balance using the Biodex Stability System in rheumatoid arthritis patients. Clin Rheumatol 2006;25:462-7.

4. Warenczak A, Lisinski P, Huber J. Importance of the functional examination in lower extremities in patients with rheumatoid arthritis. Acta Bioeng Biomech 2014;16:103-10.

5. Hayashibara M, Hagino $H$, Katagiri $H$, Okano $T$, Okada J, Teshima R. Incidence and risk factors of falling in ambulatory patients with rheumatoid arthritis: a prospective 1-year study. Osteoporos Int 2010;21:1825-33.

6. Stanmore EK, Oldham J, Skelton DA, O'Neill T, Pilling $\mathrm{M}$, Campbell AJ, et al. Risk factors for falls in adults with rheumatoid arthritis: a prospective study. Arthritis Care Res (Hoboken) 2013;65:1251-8.

7. Stanmore EK, Oldham J, Skelton DA, O'Neill T, Pilling M, Campbell AJ, et al. Fall incidence and outcomes of falls in a prospective study of adults with rheumatoid arthritis. Arthritis Care Res (Hoboken) 2013;65:737-44.

8. Armstrong C, Swarbrick CM, Pye SR, O'Neill TW. Occurrence and risk factors for falls in rheumatoid arthritis. Ann Rheum Dis 2005;64:1602-4.

9. Brenton-Rule A, Mattock J, Carroll M, Dalbeth N, Bassett S, Menz HB, et al. Reliability of the TekScan MatScan ${ }^{\circledR}$ system for the measurement of postural stability in older people with rheumatoid arthritis. J Foot Ankle Res 2012;5:21.

10. Ekdahl C, Andersson SI, Svensson B. Muscle function of the lower extremities in rheumatoid arthritis and osteoarthrosis. A descriptive study of patients in a primary health care district. $\mathrm{J}$ Clin Epidemiol 1989;42:947-54.
11. Stel VS, Smit JH, Pluijm SM, Lips P. Balance and mobility performance as treatable risk factors for recurrent falling in older persons. $J$ Clin Epidemiol 2003;56:659-68.

12. Brenton-Rule A, Dalbeth N, Menz HB, Bassett S, Rome $\mathrm{K}$. Foot and ankle characteristics associated with falls in adults with established rheumatoid arthritis: a crosssectional study. BMC Musculoskelet Disord 2016;17:22.

13. Brenton-Rule A, Dalbeth N, Menz HB, Bassett S, Rome K. Are Foot and Ankle Characteristics Associated With Falls in People With Rheumatoid Arthritis? A Prospective Study. Arthritis Care Res (Hoboken) 2017;69:1150-5.

14. Morpeth T, Brenton-Rule A, Carroll M, Frecklington M, Rome K. Fear of falling and foot pain, impairment and disability in rheumatoid arthritis: a case-control study. Clin Rheumatol 2016;35:887-91.

15. Bugdayci D, Paker N, Rezvani A, Kesiktas N, Yilmaz $\mathrm{O}$, Sahin M, et al. Frequency and predictors for falls in the ambulatory patients with rheumatoid arthritis: a longitudinal prospective study. Rheumatol Int 2013;33:2523-7.

16. Stanmore EK, Oldham J, Skelton DA, O'Neill T, Pilling $\mathrm{M}$, Campbell AJ, et al. Risk factors for falls in adults with rheumatoid arthritis: a prospective study. Arthritis Care Res (Hoboken) 2013;65:1251-8.

17. Göksel Karatepe A, Günaydin R, Adibelli ZH, Kaya T, Duruöz E. Foot deformities in patients with rheumatoid arthritis: the relationship with foot functions. Int $\mathrm{J}$ Rheum Dis 2010;13:158-63.

18. Platto MJ, O'Connell PG, Hicks JE, Gerber LH. The relationship of pain and deformity of the rheumatoid foot to gait and an index of functional ambulation. $J$ Rheumatol 1991;18:38-43.

19. İnanır A, Okan S, Ylldırım E. Postural stability and fall risk in rheumatoid arthritis. Cukurova Med J 2013;38:72-7.

20. Yoshida T, Ikemiyagi F, Ikemiyagi $Y$, Tanaka $T$, Yamamoto M, Suzuki M. The dominant foot affects the postural control mechanism: examination by body tracking test. Acta Otolaryngol 2014;134:1146-50.

21. Ekdahl C. Postural control, muscle function and psychological factors in rheumatoid arthritis. Are there any relations? Scand J Rheumatol 1992;21:297-301.

22. Häkkinen $\mathrm{A}$, Kautiainen $\mathrm{H}$, Hannonen $\mathrm{P}$, Ylinen J, Mäkinen H, Sokka T. Muscle strength, pain, and disease activity explain individual subdimensions of the Health Assessment Questionnaire disability index, especially in women with rheumatoid arthritis. Ann Rheum Dis 2006;65:30-4.

23. Böhler C, Radner H, Ernst M, Binder A, Stamm T, Aletaha $\mathrm{D}$, et al. Rheumatoid arthritis and falls: the influence of disease activity. Rheumatology (Oxford) 2012;51:2051-7.

24. Ben Moussa Zouita A, Majdoub O, Ferchichi H, Grandy K, Dziri C, Ben Salah FZ. The effect of 8-weeks proprioceptive exercise program in postural sway and isokinetic strength of ankle sprains of Tunisian athletes. Ann Phys Rehabil Med 2013;56:634-43. 